\title{
Addressing and manipulation of individual hyperfine states in cold trapped molecular ions and application to $\mathrm{HD}^{+}$frequency metrology
}

\author{
U. Bressel, A. Borodin, J. Shen, M. Hansen, I. Ernsting, S. Schiller \\ Institut für Experimentalphysik, Heinrich-Heine-Universität Düsseldorf, Universitätsstr. 1, 40225 Düsseldorf, Germany
}

\begin{abstract}
Advanced techniques for manipulation of internal states, standard in atomic physics, are demonstrated for a charged molecular species for the first time. We address individual hyperfine states of ro-vibrational levels of a diatomic ion by optical excitation of individual hyperfine transitions, and achieve controlled transfer of population into a selected hyperfine state. We use molecular hydrogen ions $\left(\mathrm{HD}^{+}\right)$as a model system and employ a novel frequency-comb-based, continuous-wave $5 \mu \mathrm{m}$ laser spectrometer. The achieved spectral resolution is the highest obtained so far in the optical domain on a molecular ion species. As a consequence, we are also able to perform the most precise test yet of the ab-initio theory of a molecule.
\end{abstract}

Cold trapped molecules [1, 2] currently represent an intense field of activity relying on sophisticated methods of molecule production, translational and internal cooling, spectroscopy and sensitive detection. Many applications, such as chemical reaction studies [3, 4], tests of molecular quantum theory [5], fundamental physics [6, 7] and quantum computing [8] would benefit strongly from the availability of advanced manipulation techniques, already standard in atomic physics. These are not straightforward for molecules, and for charged molecules have not been demonstrated yet. Production methods for molecular ions (usually by electron impact ionization) and, if heteronuclear, their interaction with the black-body radiation of the surrounding vacuum chamber, usually lead to significant population of a substantial number of internal states. A first, important step in the manipulation of internal states of molecular ions is population transfer between rotational states (heteronuclear molecules usually being cold vibrationally, i.e. are all in the $v=0$ ground vibrational state). It has been demonstrated that a significant fraction (ca. $75 \%$ ) of an ensemble of diatomic molecular ions can be pumped into the vibrational and rotational ground level $(v=0, N=0)$ [9, 10, see Fig. 1.

For a general diatomic molecule, however, this pumping is usually not capable of preparing molecules in a single quantum state, because spin interactions generate a hyperfine structure with several states in each ro-vibrational level. For example, a diatomic molecule with one unpaired electron $\left(s_{e}=1 / 2\right)$, and nuclei with nuclear spins $I_{1}=1 / 2, I_{2}=1$ (such as $\mathrm{HD}^{+}$) has 4 hyperfine states in zero magnetic field if the rotational angular momentum $N=0$, but 10 if $N=1$, and 12 if $N \geq 2$, see Fig. 2 a. The ability to address selectively molecules in one particular hyperfine state (or even in a single quantum state with a particular magnetic quantum number $J_{z}$ ) and to transfer molecules from one hyperfine state to another are clearly important tools of a molecular quantum toolbox that can be part of a full quantum state preparation procedure.

Complicating the addressing, the number of strong transitions between two given ro-vibrational levels $(v, N),\left(v^{\prime}, N^{\prime}\right)$ is equal to the larger of the two numbers of hyperfine states, i.e. potentially high, and with only small differences in transition frequency. Fig. $2 \mathrm{~b}$ shows as an example the case of the fundamental vibrational transition $(v=0, N=0) \rightarrow\left(v^{\prime}=1, N^{\prime}=1\right)$ in $\mathrm{HD}^{+}$, where 10 strong transitions occur over a range of about $60 \mathrm{MHz}([11$, 12]). Addressing a single hyperfine state in a multi-spin molecule thus requires a spectroscopy that can resolve individual "hyperfine" lines in the spectrum.

In this work, our approach is based on one-photon laser excitation of the fundamental vibrational transition $(0,0) \rightarrow$ $(1,1)$ at the wavelength $\lambda_{f}$, see Fig. 1. The relatively low transition frequency, $\lambda_{f}>2.5 \mu \mathrm{m}$ for diatomics, in combination with the low secular kinetic energy $k_{B} T_{\text {sec }}$ achievable by sympathetic cooling, yields a Doppler broadening $\Delta \nu_{D}$ of the transitions that is smaller than many line spacings. This provides the desired quantum state selectivity for addressing some of the hyperfine states, using strong transitions. For our test case $\mathrm{HD}^{+}, \lambda_{f}=5.1 \mu \mathrm{m}, T_{\text {sec }} \simeq 10 \mathrm{mK}$, $\Delta \nu_{D} \simeq 3 \mathrm{MHz}$. Additionally, excitation of weak transitions (which violate the approximate selection rules $\Delta F=0$, $\Delta S=0$, see Fig. 2 b), provides selectivity for all hyperfine states, since for these transitions the frequency spacings are larger. Compared to the use of a pure rotational excitation $(0,0) \rightarrow(0,1)$ or a microwave transition within a ro-vibrational level, the use of a vibrational transition has the advantage that the excitation may be followed by a much faster spontaneous decay, either back into the ground ro-vibrational level (rate approx. $6 \mathrm{~s}^{-1}$, here) or into the (relatively long-lived) rotational level $\left(v^{\prime \prime}=0, N^{\prime \prime}=2\right)$ (rate approx. $12 \mathrm{~s}^{-1}$, here). This allows reasonably rapid pumping of the molecule (possibly after repeated absorption and spontaneous emission events) into another long-lived state, a necessary condition for efficient quantum state preparation, as shown below.

The experiment is performed on ensembles of $\mathrm{HD}^{+}$ions trapped in a linear quadrupole radio-frequency trap (14.2 MHz), sympathetically translationally cooled by co-trapped, laser-cooled Beryllium atomic ions [13] and rotationally cooled by lasers. Our laser system consists of four subsystems: the $\lambda_{f}=5.1 \mu \mathrm{m}$ laser spectrometer referenced to an atomic frequency standard [14] (see Supplementary materials), a reliable, frequency-stabilized fiber-laser-based 
$313 \mathrm{~nm}$ laser for cooling of Beryllium ions [15, a rotational cooling laser system (a $\lambda_{p}=5.4 \mu \mathrm{m}$ quantum cascade laser and, for part of the measurements, a $\lambda_{p}^{\prime}=2.7 \mu \mathrm{m}$ diode laser), and a pair of lasers $\left(\lambda, \lambda^{\prime}\right)$ for resonance-enhanced multi-photon dissociation (REMPD), see Fig. 1. Rotational cooling [9] is a crucial tool here, as it significantly increases the fractional population of molecules in the lower ro-vibrational level $(v=0, N=0)$, from ca. $10 \%$ to 60 $75 \%$. The possibility provided by our laser system to measure the $\mathrm{HD}^{+}$fundamental vibrational transition frequency $f=c / \lambda_{f}$ also allows us to perform a precise comparison with the ab-initio theory of the molecular hydrogen ion.

Hyperfine-resolved ro-vibrational transitions are induced by the $\lambda_{f}=5.1 \mu \mathrm{m}$ laser tuned precisely to individual transitions. In order to show that this is actually achieved, we detect (without hyperfine state selectivity) the population of the goal vibrational level $\left(v^{\prime}=1, N^{\prime}=1\right)$, by $1+1^{\prime}$ resonance-enhanced multi-photon dissociation (REMPD) [16], see Fig. 1. Together, this represents a three-photon (1+1'+1") REMPD process. The reduction of the $\mathrm{HD}^{+}$number by the REMPD process is our spectroscopic signal [16.

Figure 3 shows the obtained hyperfine-state resolved spectrum. All theoretically predicted and addressed hyperfine transitions were observed; only the (nominally strong) transition S4 is barely detected, for unknown reason. Transition W4, which originates from a lower hyperfine state with only a small fractional population $(J=0$, thus statistically containing only $5-6 \%$ of all molecules), could only be made clearly evident using a preceding hyperfine pumping

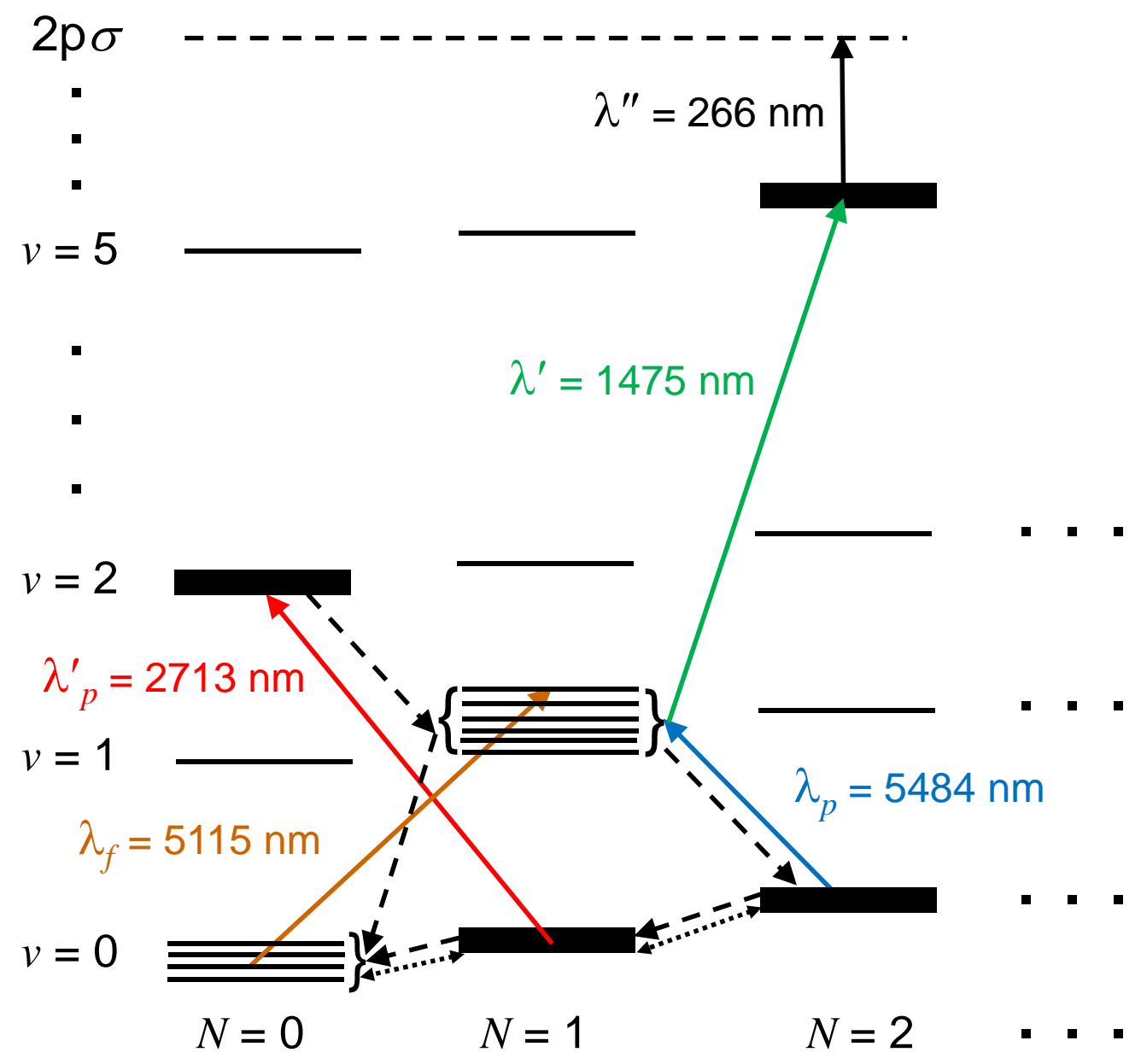

Figure 1: Schematic energy level scheme of $\mathrm{HD}^{+}$with transitions relevant to this work. Hyperfine structure is shown schematically only for the $(v=0, N=0)$ and $(1,1)$ levels as lines, but is implied for all other levels as well (thick bars). Full lines: laser-induced transitions, dashed lines: some relevant spontaneous emission transitions; dotted lines: some black-body induced transitions. The spectrally narrow wave $\lambda_{f}$ selectively excites molecules from a particular hyperfine state $(v=0, N=$ $0, F, S, J)$ to a single hyperfine state $\left(1,1, F^{\prime}, S^{\prime}, J^{\prime}\right)$. Quantum state preparation is performed by irradiating alternatingly the appropriately tuned wave $\lambda_{f}$ and $\lambda_{p}$, in conjunction with spontaneous emission from the level $(1,1)$. Resonant laser radiation at $\lambda^{\prime}$ and nonresonant radiaton at $\lambda^{\prime \prime}$ is used to detect that hyperfine-state-selective excitation to the $(1,1)$ level has occurred, by transferring the excited molecules to the electronically excited molecular state $2 \mathrm{p} \sigma$ from which they dissociate. Initially, rotational cooling is performed by radiation at $\lambda_{p}$ and $\lambda_{p}^{\prime}$. The level energy differences are not to scale. The waves at $\lambda^{\prime}, \lambda^{\prime \prime}, \lambda_{p}, \lambda_{p}^{\prime}$ have large spectral linewidths and do not excite hyperfine state-selectively. 

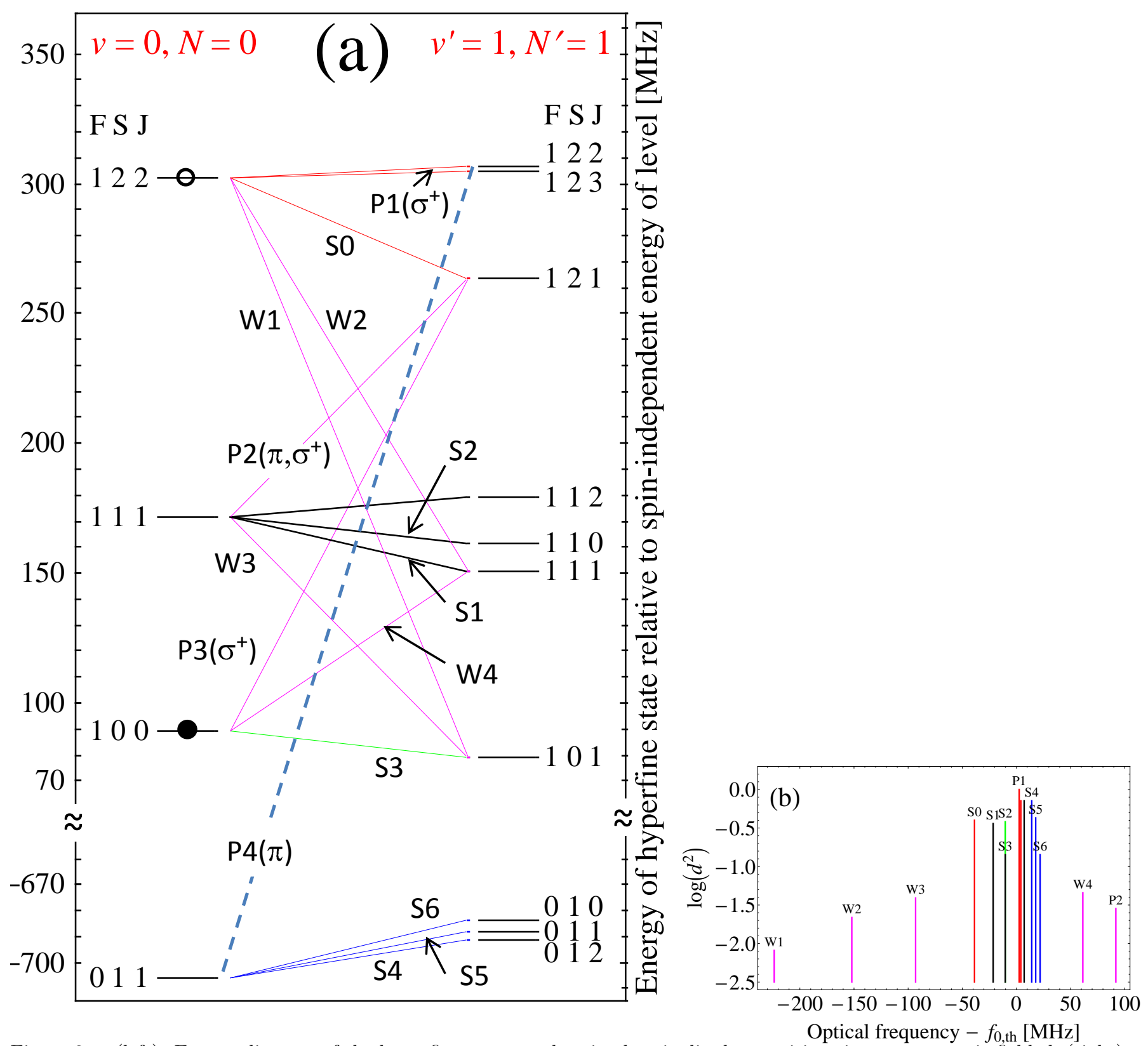

Figure 2: a (left): Energy diagram of the hyperfine states and main electric-dipole transitions in zero magnetic field. $\mathrm{b}$ (right): Stick spectrum of the transitions (in zero field; values of the squared transition moment $d^{2}$ are normalized to the strongest transition). The states are labeled by the quantum numbers $(F, S, J)$. Weak transitions are shown in pink [12. Very weak transitions are not shown, except for P4 (dashed). The "spin-independent" transition frequency $f_{0, t h}$ is the value if nuclear and electron spin were zero. S0, S1, S2, S3, S4, S5, S6, W1, W2, W3, W4, P2 are transitions studied here ("W, S, P" mean "weak", "strong", "pumping", respectively). All were observed except S4. W1, W3 are the transitions used here to achieve population transfer from the hyperfine states $\left(v=0, N=0, F=1, S=2, J=2, J_{z}\right)$ (empty circle) and $\left(0,0,1,1,1, J_{z}^{\prime}\right)$ into the hyperfine state $\left(0,0,1,0,0, J_{z}^{\prime \prime}\right)$ (filled circle). P1, P2, P3, P4 are proposed optical pumping transitions (with indicated polarizations) for preparation of the molecule in the single quantum state $\left(0,0,1,2,2, J_{z}=+2\right)$ (one of the Zeeman states in the open circled hyperfine state).

step (see below). Each of the 4 hyperfine states of the lower level was selectively addressed, and 5 of the 12 upper level hyperfine states were selectively populated. We also observed the line at $-10.2 \mathrm{MHz}$, which contains two nearly coinciding transitions S2, S3, but originating from different ground hyperfine states. The remaining strong transitions (including the line marked P1 in Fig. 20 were also observed, but their small spacing prevents complete resolution, and they are not reported in the Figure 3 .

We demonstrate hyperfine state manipulation by optical pumping of individual hyperfine state populations into a 


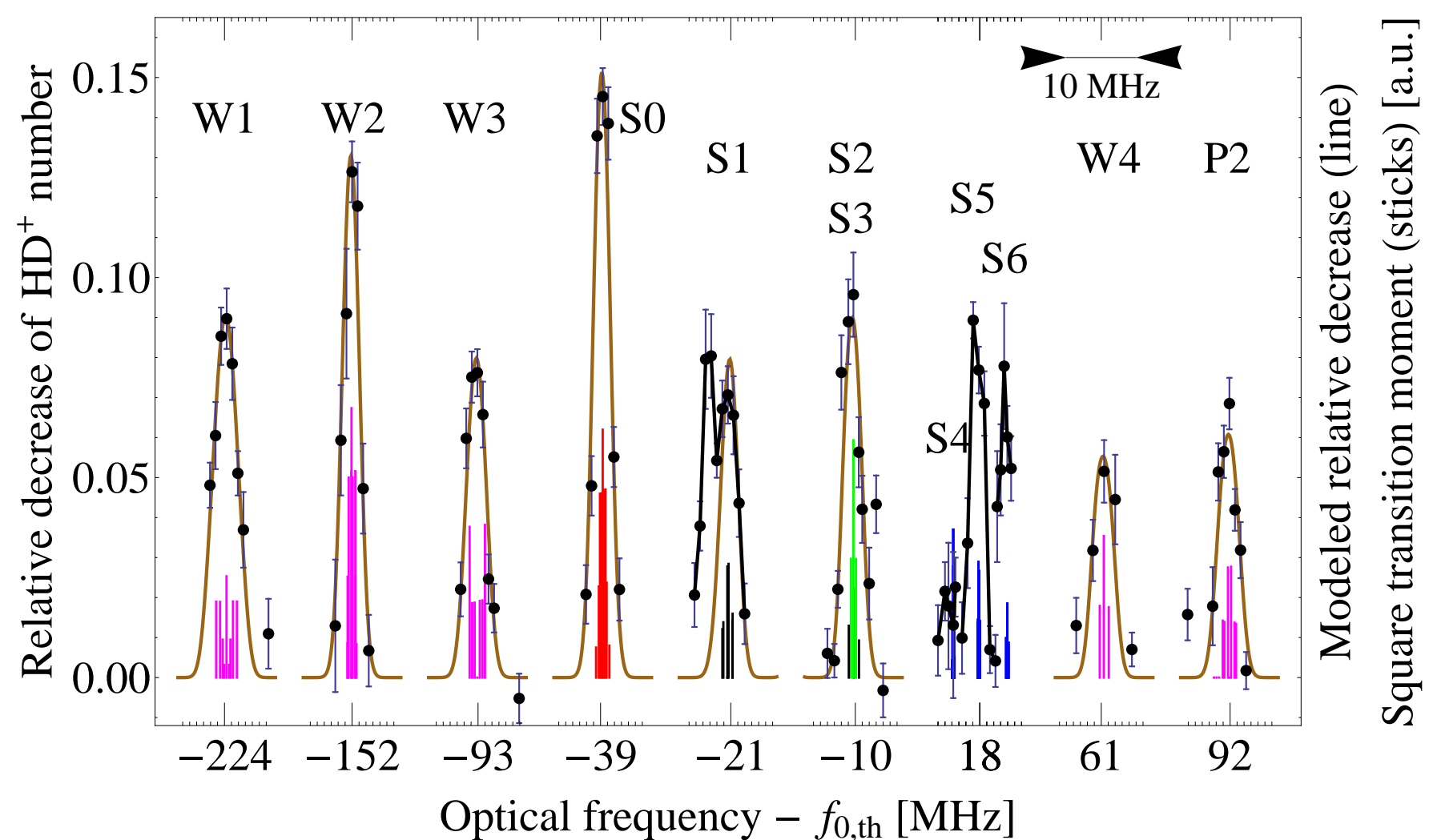

Figure 3: Observed hyperfine spectrum of the $(v=0, N=0) \rightarrow(1,1)$ fundamental ro-vibrational transition in cold trapped $\mathrm{HD}^{+}$ions. The effective intensity times irradiation duration product of the $5.1 \mu \mathrm{m}$ radiation varied from line to line, and was adapted to avoid saturation. Brown lines are the result of fitting $f_{0, \exp }$ and the individual line amplitudes for $9.5 \mathrm{mK}$ temperature, and an average magnetic field of $0.8 \mathrm{G}$. The sticks are for illustration purpose and show the theoretical squared transition dipole moments for the Zeeman components at $0.8 \mathrm{G}$, assuming exciting radiation polarized at 45 degrees to the magnetic field. They are scaled by different factors for presentation purpose. Color coding is as in Fig. 2 S4, S6 were taken at high intensity-irradiation time product, S5 at a lower value. The side peak of S1 is probably due to an ion micromotion sideband of S2/S3. The W4 line required hyperfine state optical pumping for its detection (see Fig. 4).

goal state (filled circle in Fig. 2p). As a goal state we choose $\left(v=0, N=0, F=1, S=0, J=0, J_{z}=0\right)$ which is non-degenerate $(J=0)$ and thus a single quantum state. After rotational cooling, we apply the following sequence twice: W1 line $(3 \mathrm{~s})$, rotational re-pumping $\left(\lambda_{p}\right.$ and $\lambda_{p}^{\prime}$ simultaneously for $\left.5 \mathrm{~s}\right)$, W3 line $(3 \mathrm{~s})$. A final $10 \mathrm{~s}$ of rotational re-pumping is performed before the spectroscopic excitation. The W1 and W3 transitions excite population from two initial hyperfine states $\left(0,0,1,2,2, J_{z}\right),\left(0,0,1,1,1, J_{z}^{\prime}\right)$ (without $J_{z}$ selectivity) into the same hyperfine state $\left(1,1,1,0,1, J_{z}^{\prime \prime}\right)$. This state has dominant spontaneous decay to the goal state (green line S3 in Fig. 22. We find clear evidence that this hyperfine state preparation is taking place by observing the transition W4 starting from the goal state by REMPD, see Fig. 4. This transition is not observable in our experiment without the preparation procedure, since then the population in the lower hyperfine state is too low.

Our hyperfine-state resolved spectrum represents the highest-resolution optical spectrum of any molecular ion so far [5, 17, 18. This enables an accurate comparison of experimental frequencies with ab-initio theory. We obtain two hyperfine state separations in the ground state, $\Delta f_{0,0, a}=(E(0,0,1,2,2)-E(0,0,1,1,1)) / h$ and $\Delta f_{0,0, b}=(E(0,0,1,1,1)-E(0,0,1,0,0)) / h$, from the measured transition frequency combinations $f(S 1)-f(W 2)$, $f(W 3)-f(W 1), f(P 2)-f(S 0)$, and from $f(W 4)-f(S 1), f(P 3)-f(P 2)$, respectively. In addition, two excited state splittings, $\Delta f_{1,1, c}=(E(1,1,1,2,1)-E(1,1,1,1,1)) / h$ and $\Delta f_{1,1, d}=(E(1,1,1,1,1)-E(1,1,1,0,1)) / h$ are similarly obtainable by suitable frequency combinations. A fit of these hyperfine state separations to the data (fitting also the spin-independent frequency) yields agreement with the ab-initio results $\left(\Delta f_{0,0, a}, \Delta f_{0,0, b}, \Delta f_{1,1, c}, \Delta f_{1,1, d}\right)_{t h}=$ (130.60(1), 82.83(1), 113.33(1), 71.68(1)) MHz [11, 19], with deviations (exp. - theory) of $(-0.22(0.13), 0.28(0.38)$, $-0.13(0.15), 0.27(0.19)) \mathrm{MHz}$. The two measured hyperfine separations of the ground state also allow determining the two hyperfine constants $E_{4}(0,0), E_{5}(0,0)[11$ which fully describe the hyperfine structure of the ground state [12]. Our fit yields $\left(E_{4}(0,0), E_{5}(0,0)\right)=(906(17), 142.33(25)) \mathrm{MHz}$, whereas the theory values are $(925.38(1), 142.29(1)) \mathrm{MHz}$ 


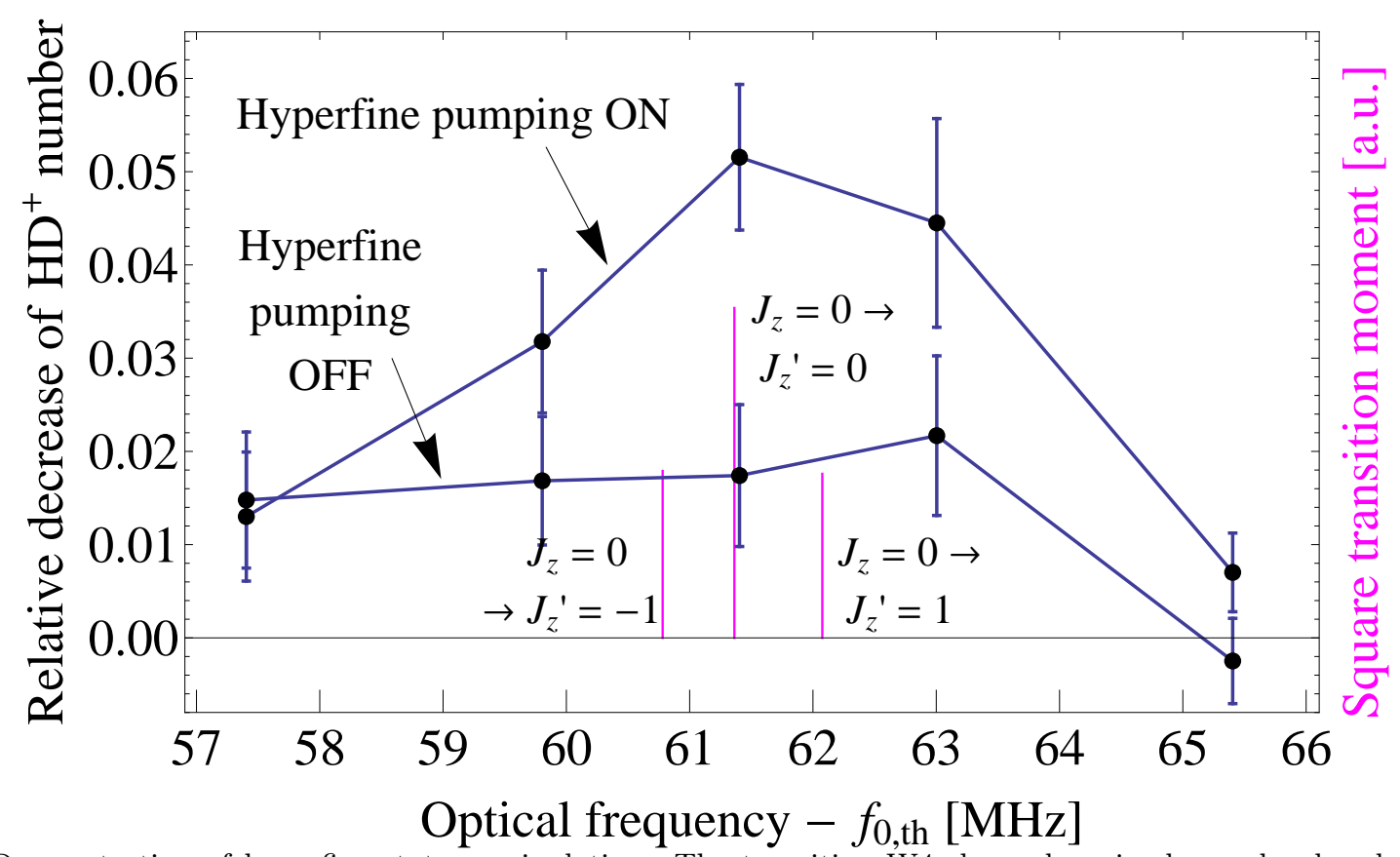

Figure 4: Demonstration of hyperfine state manipulation. The transition W4 shown here is observed only when hyperfine optical pumping is implemented. This transition represents the excitation from a single quantum state, $\left(0,0,1,0,0, J_{z}=0\right)$. Data shown was taken alternating measurements preceded by hyperfine optical pumping (upper data points joined by line) and not (lower data points). The intensity of the $5.1 \mu \mathrm{m}$ laser was set to its maximum both during hyperfine pumping on the W1 and W3 transitions and subsequent detection of the W4 transition. Irradiation time on the W4 transition was 3 s. Rotational cooling by the $2.7 \mu \mathrm{m}$ and $5.5 \mu \mathrm{m}$ laser was used. The zero level corresponds to the relative decrease measured when the $5.1 \mathrm{\mu m}$ spectroscopy laser was blocked. The three sticks show, for illustration purposes, the theoretical transition frequencies and strengths in a $0.8 \mathrm{G}$ magnetic field and radiation polarized at 45 degree to the magnetic field. The shift of the central component is $-0.05 \mathrm{MHz}$ relative to the zero-field frequency.

[11, 19].

Assuming instead that the hyperfine energies are given by the theoretical values (this assumption being strenghtened by the agreement of hyperfine theory and experiment for large- $v$ levels [11, 20]), we can fit an overall frequency correction to the spectra $\mathrm{W} 1, \mathrm{~W} 2, \mathrm{~W} 3, \mathrm{~S} 0, \mathrm{~S} 1, \mathrm{~S} 2+\mathrm{S} 3, \mathrm{~W} 4, \mathrm{P} 2$. We obtain the spin-independent frequency $f_{0, \exp }=$ $58605052.00 \mathrm{MHz}$, with combined statistical and systematic error of $0.064 \mathrm{MHz}$ (see Supplemental Materials). The theoretical value is $f_{0, t h}=58605052.139(11)(21) \mathrm{MHz}$, where the first error is due to the uncertainty of the fundamental constants and the second is the theoretical error in the evaluation of the QED contributions [19, 21, 22]. The difference between exprimental and theoretical resuls is -2.0 times the combined theoretical plus experimental error. The relative experimental uncertainty of $1.1 \times 10^{-9}$ represents the most accurate test of molecular theory to date. In particular, our measurement is the first molecular measurement sufficiently accurate to be explicitly sensitive to the QED contributions of order $\alpha^{5}$ (relative to the nonrelativistic contribution to the transition frequency), calculated as $0.109(21) \mathrm{MHz}$ for the transition studied here.

Based on the technique demonstrated here, we can propose a realistic optical pumping procedure for preparing most of the population in a single quantum state $\left(v, N, F, S, J, J_{z}\right)$, i.e. with well-defined projection of the total angular momentum. Under typical conditions, the relative statistical occupation of any individual quantum state in $(v=0, N=0)$ is only $\simeq(1 / 12) \times(60 \%-75 \%) \simeq 5 \%-6 \%$ under rotational cooling by a single laser $\left(\lambda_{p}\right)$ or two lasers $\left(\lambda_{p}, \lambda_{p}^{\prime}\right)$. Exciting sequentially the four transitions $\mathrm{P} 4[(0,0,0,1,1) \rightarrow(1,1,1,2,2)], \mathrm{P} 3, \mathrm{P} 2, \mathrm{P} 1[(0,0,1,2,2) \rightarrow$ $(1,1,1,2,3)]$ in a weak magnetic field and with polarizations chosen as indicated in Fig. 22 a will cause transfer of the population of all Zeeman quantum states of $(0,0)$ to the single Zeeman quantum state $\left(0,0,1,2,2, J_{z}=+2\right)$, via spontaneous emission processes from $\left(1,1,1,2, J^{\prime}=\{1,2\}, J_{z}^{\prime}\right)$, which dominantly occur on strong transitions (red lines in Fig. 2 a). These excitations should be interleaved with rotational cooling (lasers $\lambda_{p}, \lambda_{p}^{\prime}$ ), which also serves as repumper following spontaneous decay into $\left(v^{\prime \prime}=0, N^{\prime \prime}=2\right)$. The optical pumping procedure should take a few ten seconds and lead to $60 \%$ - $70 \%$ fractional population in the goal state.

In summary, we have shown that it is possible to address and prepare individual hyperfine states in cold, trapped diatomic molecular ions even in presence of a complex spin structure. A mid-infrared laser spectrometer controlled by an atomic standard-referenced frequency comb, and sufficiently low ion kinetic energies were two important require- 
ments. The observed, Doppler-limited, transition linewidths $(3 \mathrm{MHz})$ are the lowest obtained to date on a molecular ion species in the optical domain (note that they scale as (molecule mass) ${ }^{-1 / 2}$ ). We also observed, for the first time to our knowledge, weakly allowed hyperfine transitions using optical excitation. As one application, we were able to directly determine the population fraction of molecules in particular hyperfine states. The largest value we found was $19 \%$, clearly indicating the effectiveness of our rotational cooling. We also demonstrated excitation of a transition from a single quantum state. Since our test molecule $\mathrm{HD}^{+}$is the simplest heteronuclear molecule and is excited from the ro-vibrational ground state, this study represents the first precision measurement of the most fundamental electric-dipole allowed ro-vibrational transition of any molecule [17]. A comparison of theory with experiment showed that (i) the hyperfine energies of small- $v, N$ ro-vibrational levels agree within deviations of less than $0.3 \mathrm{MHz}$ and (ii) the spin-independent energy agrees within 2 times the error of $1.1 \times 10^{-9}$.

ACKNOWLEDGEMENT. We are grateful to B. Roth, T. Schneider, A. Yu. Nevsky and S. Vasilyev for help and support, and to V. Korobov for important discussions. Funding was provided by DFG project Schi 431/11-1.

[1] R. Krems, B. Friedrich, W.C. Stwalley, eds., Cold Molecules (CRC Press, 2009)

[2] I.W.M. Smith, ed., Low Temperatures and Cold Molecules (World Scientific Publishing, 2008)

[3] B. Roth, et al. , Phys. Rev. A 73, 042712 (2006);

[4] P. F. Staanum, et al. , Phys. Rev. Lett. 100, 243003 (2008)

[5] J.C.J. Koelemeij, B. Roth, A. Wicht, I. Ernsting and S. Schiller, Phys. Rev. Lett. 98, 173002 (2007)

[6] S. Schiller and V. Korobov, Phys. Rev. A 71, 032505 (2005)

[7] H. Müller, et al. , Phys. Rev. A 70, 076004 (2004)

[8] D. DeMille, Phys. Rev. Lett. 88, 067901 (2002)

[9] T. Schneider, et al. , Nature Physics 6, 275 (2010)

[10] P. Staanum, et al., Nature Physics 6, 271 (2010)

[11] D. Bakalov, et al., Phys. Rev. Lett. 97, 243001 (2006)

[12] D. Bakalov, et al., J. Phys. B: At. Mol. Opt. Phys. 44, 025003 (2011)

[13] P. Blythe, et al. , Phys. Rev. Lett. 95, 183002 (2005)

[14] U. Bressel, I. Ernsting, and S. Schiller, Opt. Lett. 37, 918 (2012).

[15] S. Vasilyev, et al. , Appl. Phys. B 103, 27 (2011)

[16] B. Roth, et al., Phys. Rev. A 74, 040501-4 (2006)

[17] The $(0,1) \leftrightarrow(1,0)$ transition has been measured in an ion beam experiment, with a linewidth $\geq 7 \mathrm{MHz}$ and a frequency uncertainy $\simeq 21 \mathrm{MHz}$, W.H. Wing, et al. , Phys. Rev. Lett. 36, 1488 (1976); J.J. Spezeski, Ph.D. dissertation, Yale University, 1977

[18] H. C. Miller, M. Al-Za'al, J. W. Farley, Phys. Rev. Lett. 58, 2031 (1987)

[19] V. I. Korobov, private communication (2010)

[20] A. Carrington et al., Mol. Phys. 72, 735 (1991)

[21] V.I. Korobov, Phys. Rev. A 74, 052506 (2006)

[22] V.I. Korobov, Phys. Rev. A 77, 022509 (2008) 


\section{Supplemental Materials}

\section{General features of hyperfine structure}

We consider a diatomic molecule with total electron spin $s_{e}$, nuclei with nuclear spins $I_{1}, I_{2}$, in a rotational level $N$. The spin degeneracy is lifted (only partially in zero magnetic field) into a number of hyperfine states by the electron spin - nuclear spin interaction $\left(\sim \mathbf{s} \cdot \mathbf{I}_{\mathbf{i}}\right)$ and/or the electron spin - rotation interaction $(\sim \mathbf{s} \cdot \mathbf{N})$. In $\mathrm{HD}^{+}$, the (approximate) quantum numbers $(F, S, J)$ correspond to the couplings $\mathbf{F}=\mathbf{s}+\mathbf{I}_{\mathbf{p}}, \mathbf{S}=\mathbf{F}+\mathbf{I}_{\mathbf{d}}, \mathbf{J}=\mathbf{S}+\mathbf{N}$. The electron spin - nuclear spin interactions determine the main splittings in a given ro-vibrational level, resulting in 4 singlets (if $N=0$ ) or in 4 multiplets (if $N \neq 0$ ). In the latter case, the electron-spin rotation interaction determines the splitting strength within the multiplets [11].

The line splittings in the transition spectrum, Fig. 2 b, arise because in the lower and upper levels (i) the strengths of the electron spin-nuclear spin interactions and of the electron spin - rotation interaction differ, and (ii) the rotational angular momenta $N, N^{\prime}$ differ. The density of lines in the spectrum is essentially independent of whether the transition is a fundamental vibrational transition, an overtone transition $\left(\Delta v=v^{\prime}-v>1\right)$ (e.g. [16]), or a pure rotational transition $(\Delta v=0)$.

\section{2. $\quad 5.1 \mu \mathrm{m}$ laser spectrometer}

The spectroscopy radiation was generated by frequency-mixing in a nonlinear optical crystal two near-infrared lasers which are individually referenced to a conventional frequency comb [14]. The generated wavelength, $5.1 \mu \mathrm{m}$, extends the coverge provided so far by sources with absolute frequency measurement capability [1, 2].

We use two continuous-wave lasers, a Nd:YAG laser $(1064 \mathrm{~nm}$, ca. $6 \mathrm{~W})$ stabilized to a Doppler-free resonance in molecular iodine via its second-harmonic wave at $532 \mathrm{~nm}$, and a $1344 \mathrm{~nm}$ home-built external cavity quantum-dot diode laser (QD-ECDL). The main part of the $1344 \mathrm{~nm}$ wave is resonantly enhanced in a ring resonator containing a periodically poled $\mathrm{MgO}: \mathrm{LiNbO}_{3}$ crystal with poling period $25.4 \mu \mathrm{m}$, appropriate for difference frequency generation of $5.1 \mu \mathrm{m}$ radiation. The $1064 \mathrm{~nm}$ wave is focused and overlapped with the $1344 \mathrm{~nm}$ wave in the crystal, but not resonated. Although the crystal strongly absorbs the generated $5.1 \mu \mathrm{m}$ radiation, an output power of up to $0.1 \mathrm{~mW}$ is generated. Small fractions of the 1064 and $1344 \mathrm{~nm}$ waves are sent via an unstabilized single-mode optical fiber to another laboratory containing the femtosecond Ti:sapphire frequency comb, where both frequencies are measured. The $1344 \mathrm{~nm}$ laser is actively frequency-stabilized to a mode of the frequency comb. The $1064 \mathrm{~nm}$ wave frequency is continuously measured by the comb. The comb itself is stabilized to a hydrogen maser or ultra-stable quartz oscillator, both referenced to GPS.

Tunable, frequency-stable $5.1 \mu \mathrm{m}$ radiation is produced by amplitude modulating the diode laser output wave with an integrated-optic modulator at a variable radio-frequency $\Omega$ before sending the wave to the resonator. This generates two sidebands, and the resonator length is stabilized to resonate one of the two, generating a single frequency at 5.1 $\mu \mathrm{m}$. By changing the modulation frequency $\Omega$, the $5.1 \mu \mathrm{m}$ radiation can be smoothly and precisely frequency-tuned over $460 \mathrm{MHz}$. We determined the spectral width of the $5.1 \mu \mathrm{m}$ radiation as $\Gamma_{f} \simeq 0.68 \mathrm{MHz}$. The frequency instability (Allan deviation) of this radiation is constant at ca. $23 \mathrm{kHz}$ for integration times $\tau$ up to $10 \mathrm{~s}$, dropping to $4 \mathrm{kHz}$ at $\tau=800 \mathrm{~s}$. Both linewidth and instability are significantly lower than the Doppler width of the $\mathrm{HD}^{+}$hyperfine transitions studied here. The absolute frequency uncertainty of the $5.1 \mu \mathrm{m}$ radiation is less than $10 \mathrm{kHz}$ when averaged over $1 \mathrm{~h}$.

\section{Procedures and analysis}

Each data point in a spectrum measurement is acquired from one loading of $\mathrm{HD}^{+}$ions into a laser-cooled $\mathrm{Be}^{+}$ion cluster. Fig. 5 shows an example.

From molecular dynamics (MD) simulation of ion cluster fluorescence images 3 , we deduce an upper limit for the secular temperature $T_{s e c} \leq 15 \mathrm{mK}$. After loading, the molecules are rotationally cooled by $\lambda_{p} \simeq 5.5 \mu \mathrm{m}$ radiation (and, for some measurements, also $\lambda_{p}{ }^{\prime}=2.7 \mu \mathrm{m}$ radiation) for $30 \mathrm{~s}$, transferring about $60 \%$ (75\%) of the molecules into the $(v=0, N=0)$ ro-vibrational ground level. This population is spread over the four hyperfine states. Then, the rotational pumping laser waves are blocked and the $5.1 \mu \mathrm{m}$ spectroscopy radiation is applied along the trap axis for 3 - $30 \mathrm{~s}$, simultaneously with a $1475 \mathrm{~nm}$ diode laser (ca. $1 \mathrm{~mW})$ driving the $\left(v^{\prime}=1, N^{\prime}=1\right) \rightarrow\left(v^{\prime \prime}=5, N^{\prime \prime}=2\right)$ overtone transition, and a $266 \mathrm{~nm}$ laser (ca. $25 \mathrm{~mW}$ ) that dissociates the molecules in the latter state.

The number of trapped $\mathrm{HD}^{+}$ions is measured by excitation of their secular motion before the rotational cooling and after the (partial) photodissociation. Excitation of the secular motion heats up all ions and therefore changes 


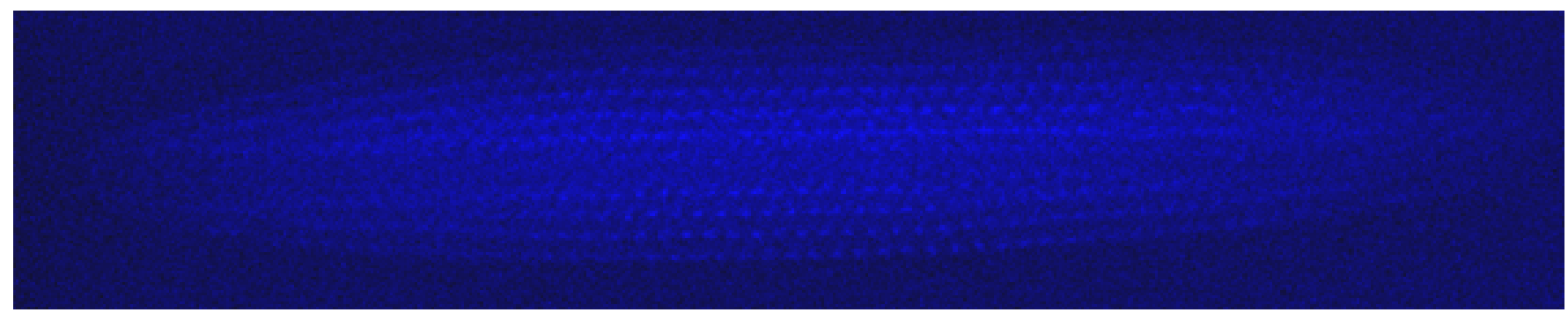

Figure 5: CCD image of a typical Beryllium ion Coulomb cluster with embedded $\mathrm{HD}^{+}$ions (darker region along the long axis), as used in the experiment.

the $\mathrm{Be}^{+}$fluorescence rate, which is detected by a photomultiplier. The relative decrease in the photomultiplier signal is the signal of interest, the excited molecule fraction. As the spectroscopy source linewidth is $0.7 \mathrm{MHz}$, we made frequency steps of typically $0.8 \mathrm{MHz}$ when scanning through the lines. One data point could be recorded on average every $3 \mathrm{~min}$. This limited the number of data points per frequency value that could be taken. We performed between 4 and 25 measurements per frequency point. Typically, one hyperfine transition required one day of measurement. Background level measurements were performed in the same way, with the $5.1 \mathrm{\mu m}$ laser blocked. Alignment of the $5.1 \mathrm{um}$ laser with the ion cluster varied from day to day, which was compensated by adjusting the power.

The typical standard deviation of the relative decay data taken at a given optical frequency (not of the mean) is 0.02. This is due primarily to the low number of $\mathrm{HD}^{+}$ions loaded into the ion cluster (a few hundred) and the low relative population in a particular hyperfine state (only a fraction $\simeq(0.6-0.75) p_{J}$, where $p_{J}=(2 J+1) / 12$ in a particular hyperfine state $J$, for $N=0$ ), which is further reduced during the REMPD phase $\left(\lambda_{p}, \lambda_{p}{ }^{\prime}\right.$ lasers off) by the competing black-body-radiation driven excitation $(v=0, N=0) \rightarrow(0,1)$. The overall small numbers lead to significant statistical fluctuations in the number of ions actually prepared into a particular initial hyperfine state after optical pumping, and in the fraction excited and dissociated. As a consistency check, we found that the maximum observed dissociated fractions (at high intensity and on resonance) did not exceed $p_{J}$. For example, for line S0, we observed $(19.4 \pm 1.4) \%$ ion number reduction, for lines $\mathrm{S} 2+\mathrm{S} 3,(16.4 \pm 2.8) \%$. Note that the values shown in Fig. 3 , are lower because there, lower intensities were used in order to avoid saturation broadening.

The smallest observed full-width-half-maximum linewidths are $\simeq 3.0 \mathrm{MHz}$. Assigning this to be due to Doppler broadening only, the value yields an upper limit of $15 \mathrm{mK}$ for the secular temperature, consistent with the MD simulations.

The observable spectrum may be modeled, including the effects of finite secular temperature, finite laser linewidth, finite population in the lower level, the effect of unresolved Zeeman splittings by the non-zero magnetic field in the ions' region [12, and black-body-radiation induced excitation to $(0,1)$. In order to simplify the analysis, in the experiment we chose laser power and irradiation times such that significant saturation and concomitant broadening of the signals was avoided, i.e. the linear absorption regime was maintained. We furthermore take into account: (i) from independent measurements we have some knowledge about the magnetic field: an upper limit of ca. $1 \mathrm{G}$ [4]; (ii) the laser linewidth does not contribute strongly to the total linewidth, and we may therefore use an effective Doppler temperature for modeling the lineshape; (iii) the overlap between the focused spectroscopy beam (ca. $0.5 \mathrm{~mm}$ waist) and the ion cloud was not constant over the time span covering all hyperfine line measurements; (iv) the effect of black-body-induced excitation $(0,0) \rightarrow(0,1)$ represents a reduction in maximum observable signal. Thus, we fit an effective product of intensity and irradiation time to each transition spectrum in order to reproduce the signal levels. We used a simplified model of the magnetic field inhomogeneity in the ions' volume. We find a good fit for an average magnetic field of $0.8 \mathrm{G}$ and $(9.5 \pm 1) \mathrm{mK}$ temperature. The statistical error for the spinless frequency $f_{0, t h}$ is obtained from a Monte Carlo simulation as $60 \mathrm{kHz}$.

\section{Systematic errors}

Due to the slow data rate of this experiment, measurements of systematics were not possible. However, the welldeveloped theory of the $\mathrm{HD}^{+}$molecule allows relying on theoretical results to estimate upper limits for various systematic errors. Those due to light shift, black-body shift [6], electric quadrupole shift [7], and Stark shift [8, 9], are theoretically estimated to be less than $10 \mathrm{kHz}$. The only potential significant effect is the Zeeman effect. The sticks in Fig. 3 , 4 show the splittings expected for the typical magnetic field value in our trap [12]. These unresolved splittings could lead to a shift of the centers of the lines but we find that the weighted mean frequency of the magnetic components of any line shifts weakly with magnetic field (e.g. less than ca. $10 \mathrm{kHz}$ for line S0 at $1 \mathrm{G}$ ). We 
modeled the influence of the imprecisely known magnetic field on the fitted spin-independent frequency and estimate an error of $13 \mathrm{kHz}$ from this effect. In order to obtain the spin-independent frequency from our measurements, we use the theoretical prediction of the hyperfine energies. The influence of their theoretical errors onto the fitted spinindependent frequency is conservatively assumed to be $10 \mathrm{kHz}$. The error of the optical frequency measurements is less than $10 \mathrm{kHz}$. Similar considerations are applied to the fit of the hyperfine splittings.

\section{Theoretical transition frequencies}

The theoretical frequency of any particular hyperfine transition arises from three contributions. The main contribution is non-relativistic, $58604301.269 \mathrm{MHz}$, determined by the solution of the Schrödinger equation 21] and whose error $(11 \mathrm{kHz})$ comes dominantly from the uncertainties of the electron-proton and proton-deuteron mass ratios $m_{e} / m_{p}$, and $m_{p} / m_{d}$ [5]. A second contribution, $750.870 \mathrm{MHz}$, is from relativistic and QED effects [22], with estimated theoretical uncertainty of $21 \mathrm{kHz}$ [19]. The sum of these two contributions is the spin-independent frequency $f_{0, t h}$. The third is the hyperfine contribution [1], e.g. $-38.685 \mathrm{MHz}$ for transition S0. Improved values for the hyperfine constants and Bethe logarithm communicated recently by V. Korobov have been used here. The influence of these improved values as compared to the published ones on the results presented here is small in comparison with the experimental uncertainties. The theoretical uncertainty of the hyperfine contributions to the transition frequencies is less than $10 \mathrm{kHz}[19$.

\section{Hyperfine state preparation}

The re-pumping suggested acts predominantly on the strong, non-spin-state changing transitions. P2 is a weak transition, P3, P4 are very weak transitions. The transition dipole moments of the latter are so small [12] that a quantum cascade laser (typical output power level $10 \mathrm{~mW}$ ) could be used for obtaining usefully large rates. Such a laser could be frequency-stabilized to a spectrometer of the type developed here, using the beat note with it as an error signal.

An additional rotational cooling laser that depletes the $(v=0, L=3)$ level by exciting from it to the $(1,2)$ or $(2,2)$ level could enable an even higher population fraction in the goal hyperfine state.

In a similar way, any other Zeeman states of the ground vibrational level could in principle be populated to a high fraction.

It is recognized that variations of the above scheme can also be used to achieve similar population fractions in individual quantum states of the $(v=0, N=1)$ level.

[1] Wavelengths shorter than $4.4 \mu \mathrm{m}$ were obtained in: P. Cancio, S. Bartalini, S. Borri, I. Galli, G. Gagliardi, G. Giusfredi, P. Maddaloni, P. Malara, D. Mazzotti and P. De Natale, Appl. Phys. B 102, 255 (2011)

[2] For wavelengths near $10.6 \mu \mathrm{m}$, see A. Amy-Klein, A. Goncharov, M. Guinet, C. Daussy, O. Lopez, A. Shelkovnikov, and C. Chardonnet, "Absolute frequency measurement of a $\mathrm{SF}_{6}$ two-photon line by use of a femtosecond optical comb and sum-frequency generation", Opt. Lett. 30, 3320 (2005)

[3] C. B. Zhang, D. Offenberg, B. Roth, M. A. Wilson, and S. Schiller, Phys. Rev. A 76, 012719 (2007)

[4] J. Shen, A. Borodin, M. Hansen, and S. Schiller, subm. to Phys. Rev. A. (2011)

[5] P.J. Mohr, B.N. Taylor, and D.B. Newell (2011), "The 2010 CODATA Recommended Values of the Fundamental Physical Constants" (Web Version 6.0). This database was developed by J. Baker, M. Douma, and S. Kotochigova. Available: http://physics.nist.gov/constants. National Institute of Standards and Technology, Gaithersburg, MD 20899

[6] J.-Ph. Karr, S. Kilic, and L. Hilico, J. Phys. B 38, 853 (2005);

[7] A. K. Bhatia and R. J. Drachman, Phys. Rev. A. 61, 032503 (2000);

[8] R. E. Moss and L. Valenzano, Mol. Phys. 100, 1527 (2002)

[9] D. Bakalov and S. Schiller, to appear in Hyperfine Interactions (2012) 